Send your letters to the Editor,

British Dental Journal,

64 Wimpole Street

London

W1G 8YS

Email bdj@bda.org

Priority will be given to letters less than 500 words long.

Authors must sign the letter, which

may be edited for reasons of space.

\section{CONVERTING CLINICS}

Sir, I would like to respond to the letter from G. A. Greenwood Oblique radiographs (BDJ 2009; 206: 221) with the solution used within the dental services of Southampton City PCT when a similar problem was encountered just over a year ago, on converting some clinics to digital radiography.

Southampton City PCT has had a long tradition of using the oblique lateral (bimolar) radiograph for assessing patients, especially small children prior to extractions under general anaesthesia. Conventional tomographic and intraoral techniques are certainly not appropriate for some patients with either severe physical, medical, morphological or learning disabilities of any age.

The oblique lateral radiograph will provide two thirds of the information that is obtained on a standard OPT for the dose equivalent of a pair of bitewing or molar periapical views (depending on sensitivity of receptor) and can be easily taken under sedation or anaesthetic.

The problem was overcome by using an OPT cassette containing a digital plate produced by Kodak Practiceworks. The plate is then easily read by their CR7400 reader which is about the size of a bread bin. The image may be viewed immediately on a computer screen, stored in the digital record for that patient and/or printed in various formats. This system allows great flexibility as it also reads small intraoral plates which are similar in size and thickness to conventional film. At installation the plates will be calibrated to the reader and X-ray source.

The OPT cassette is longer and slightly narrower than the bimolar half-plate but is very user friendly as you can position it easily. Right and left views can be taken on the same plate and there is no need to shield half the plate from radiation scatter to prevent 'fogging'.

The X-ray source is a standard $70 \mathrm{Kv}$ intraoral machine. The machine was supplied with a rectangular collimator that is exchanged for a round one for oblique lateral views only. Neither I, nor any other members of staff have managed this view satisfactorily with rectangular collimation.

This new approach to an old technique will therefore require the following equipment: OPT cassette containing a digital plate (re-usable), digital reader linked to a computer, screen and printer if required. This may take up room in an operating theatre or could be sited in an adjacent room.

Regarding printers, the dental service has had better results using a good quality inkjet printer onto matt photographic paper than with a laser printer. You can also print onto acetate if you prefer. Medical grade printers are available but were prohibitively expensive for our service. Radiographs are stored and viewed in the electronic record for the patient and only printed and forwarded if the patient is referred to another service or clinic that cannot access the record.

I hope this may be of some help.

A. Keirby, Romsey

DOI: $10.1038 /$ sj.bdj.2009.520

\section{OBLIQUE LATERALS}

Sir, I was pleased to read the letter by G. A. Greenwood (BDJ 2009; 206: 221). It was as if I had written the letter myself since I also as a Senior Dental Officer practising special care dentistry for many years have found oblique lateral radiographs very useful when looking after people with severe disabilities including those examined and treated under conscious sedation and general anaesthesia. Indeed I often wonder why this technique is not used more widely by colleagues looking after similar patients. If it were, I suspect the radiographic industry would make appropriate digital sensors and cassettes more widely available.

In the mean time I have found either using a digital DPT cassette or a digital true lateral cephalometric lateral skull (lateral ceph.) cassette to fulfil the requirement although these are a little larger than the standard wet-film cassettes I have used for oblique lateral views.

K. Dalley, Warsash

DOI: 10.1038/sj.bdj.2009.521

\section{NO ADVERSE RESULTS}

Sir, when I last checked (approximately two years ago), cassettes used for oblique lateral radiography were available by special order from Minerva Dental; films were available from Henry Schein.

The intensifying screens with these cassettes are calcium tungstate and the corresponding films are the 'blue' films. This combination is much slower than speeds achieved with rare earth screens and corresponding film.

I have used DPT cassette and film with the lateral oblique technique with no adverse results. I don't see any reason why cephalometric cassettes and film could not be used.

The typical size of the lateral oblique cassette is $15 \times 18 \mathrm{~cm}$, the cephalometric $18 \times 24 \mathrm{~cm}$ and the DPT $15 \times 30 \mathrm{~cm}$. Using either the cephalometric or DPT combinations should result in a lower dose, as both are available with rare earth screens.

Should Mr Greenwood not wish to try these alternatives and can source the 
film, I would be happy to send him a 15 $\times 18 \mathrm{~cm}$ cassette with screens, which I do not use anymore.

B. Fanning

By email

DOI: 10.1038/sj.bdj.2009.522

\section{USEFUL CONTACT}

Sir, I was also a student at the London Hospital, and still use the lateral oblique technique. I am able to obtain cassettes, intensifying screens and $13 \times 18 \mathrm{~cm}$ $\mathrm{X}$-rays. I hope this is useful.

A. Somerville

Tel: 01484859330

DOI: 10.1038/sj.bdj.2009.523

\section{LEAD POISONING}

Sir, a 31-year-old Romanian male presented to the acute surgical unit with colicky abdominal pain for three weeks, recent loss of appetite, increasing constipation and no weight loss. He is a painter/decorator and migrated to the UK one year ago.

He was anaemic, with a normal general examination. An abdominal examination was unremarkable and chest and abdomen X-rays were normal. Blood and biochemical tests showed iron deficiency anaemia and a normal kidney and liver profile.

He was treated symptomatically with little improvement. On re-examination, he was noticed to have a bluish discolouration along his gum (Fig. 1). Further enquiry about his occupation revealed a heavy involvement in paint stripping old buildings over the past eight years. The blood film showed 'basophilic stippling' and serum lead levels were high.

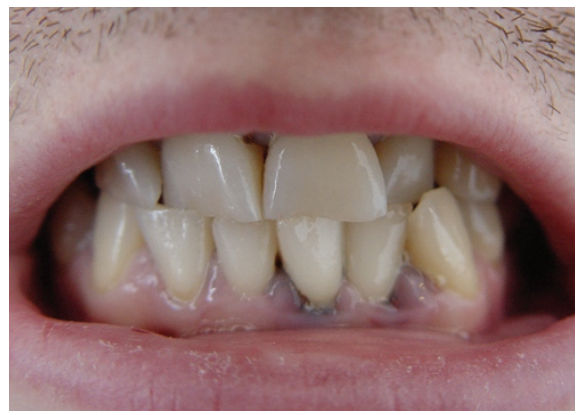

Fig. 1 Bluish discolouration along the patient's gum

A central nervous system examination was normal. He was treated with chelating agents with a good response.
Lead poisoning is a recognised occupational hazard. Lead containing paints are still encountered in old buildings and paint stripping presents a high exposure risk. Inhalation is the main route of exposure. Despite different legislation to control exposure, many Eastern European countries have no strict occupational health regulations. In other parts of the world, hazard from lead smelting and lead additives is a major problem for workers and consumers, for example: China and India (synthetic dye and lead paint and the 2007 Chinese export recalls). In the Middle East, tetra-ethyl lead is still added to petrol, posing an environmental risk.

Poisoning can be acute or chronic. Diagnosis can be difficult as symptoms and signs are non specific and present late until high blood lead levels are reached. Patients may present with nausea, vomiting, abdominal pain and constipation. CNS involvement can cause numbness and pain in the extremities, lethargy and mood disorders, muscle weakness, headache and memory loss. It can lead to miscarriage in women and reduced sperm count in men.

In 1840, Henry Burton described a bluish line in the gum following exposure to lead (Burton's line). Lead sulphide is produced by the reaction of lead with sulphur ions produced by oral bacteria which present as a narrow blue line alongside the edge of the gum in both upper and lower jaws. It is a reliable sign, but poor oral hygiene can produce a similar picture.

Laboratory findings of anaemia and punctuate basophilia 'basophilic stippling' is due to lead interference with haemoglobin synthesis and is not specific. Lead poisoning should be confirmed by biochemical testing of blood lead levels. An L-line X-ray fluorescence technique can measure cortical bone lead content.

Treatment includes preventing further exposure. Protective masks and clothing are important precautions as well as recognising jobs with high risk. Removal of lead from the body using chelating agents is the most important treatment. It is a slow process and requires monitoring of lead levels. Ethylenediaminetetraacetic acid can be given intravenously, sometimes combined with Dimercaprol (BAL). Edentate calcium disodium (EDTA calcium) and D-penicillamin are other useful agents.

A. Khalil, Perth

DOI: 10.1038/sj.bdj.2009.524

\section{WARRANTING THE EFFORT}

Sir, over the last seven years, there have only been a small number of changes in the DPF/BNF antibiotic prescribing guidelines for dental practitioners, with the removal of three indications: oralantral fistula, acute suppurative pulpitis and antibiotic prophylaxis. ${ }^{1,2}$

While the general DPF/BNF advice that 'Antibacterial drugs should only be prescribed for the treatment of dental infections on the basis of defined need' has not changed over that period, there has been a more general recognition of the need to limit the development of resistant strains by optimising prescribing patterns. ${ }^{3}$ However, recent research found that high levels of oral antibiotic prescribing in the absence of local measures persists and is of concern. ${ }^{4}$

Over a period of time I developed an antibiotic prescribing approach, which used available authoritative advice resources in order to optimise therapeutic use. This may be summarised as follows:

- Examination with particular reference to BNF advice

- Adopting a local measures approach

- Where appropriate recalling patients within 24 hours to review their condition in order to determine whether it was stable, resolving or worsening

- Providing patients with $00 \mathrm{H}$ contact advice, analgesics advice and advice to attend ActE in the event that a swelling developed which began to embarrass the airway

- Providing reassurance to patients on the clinical approach being adopted, frequently with reference to the limitations of antibiotics

- Where appropriate seeking additional professional pharmacological advice

- Immediate referral for patients with very severe dental infections, which risked embarrassing the airway (as I recall there were two such cases over 15 months both of which were 
discussed with the on-call maxillofacial clinician prior to referral).

In order to assess the outcome of this approach, a text backup document generated by keylogger software was analysed for keywords potentially relevant and relevant to acute dental infections over the period November 2007 to January 2009.

Over that period only two instances of prescribing antibiotics for a single acute dental infection were identified (I had originally believed that there were three separate episodes of such antibiotic prescribing - unfortunately the full clinical software package is not readily searchable at the text level, currently). The keywords analysed for the same period and associated number of instances are given in Table 1.

\begin{tabular}{|c|c|}
\hline & \\
\hline Keyword & Number of instances \\
\hline Pain & 1,512 \\
\hline Swelling & 752 \\
\hline Abscess & 240 \\
\hline Pulpitis & 165 \\
\hline Decompression & 78 \\
\hline Pus & 46 \\
\hline Apical periodontitis & 41 \\
\hline Trismus & 11 \\
\hline
\end{tabular}

Therefore, during that time I estimate that I treated approximately 600-800 emergency patients (dental bureau referrals, new patients and past patients). I would add that I have also not found it necessary to prescribe antibiotics in the period January-April 2009.

In England, in the period 1 April 2007 to 31 March 2008, approximately 20,000 dentists (with NHS activity) ${ }^{5}$ issued 3.7 million NHS prescriptions for antibiotics in the financial year 2007/8 at a cost of £7.89 million, in England. ${ }^{6}$ Therefore on average each NHS dentist prescribed 185 courses of antibiotics per year with a total cost of $£ 394$. The respective FTE figure per dentist may be expected to be higher and private prescribing per dentist may increase FTE figures for all dentists in England, still further. If my personal case load for acute dental infections is typical of the average practitioner, then my experiences would suggest that prescribing and associated costs could be reduced to approximately $1 \%$ of current levels. ${ }^{3}$

To be candid, such an approach to antibiotic prescribing can be time consuming and stressful, particularly where patient 'expectations' are not being met and where prescribing criteria are close to being met. Furthermore, the costs of the additional effort probably substantially outweigh the financial savings to the NHS in reduced prescription charge expenditure. However, when implemented and established, I believe the benefits to the patient and the clinician more than warrant the effort. I also believe that similar approaches will have been pursued by many colleagues and dissemination of their approaches and experiences would be of benefit to the profession.

\section{P. McCrory, Radcliffe}

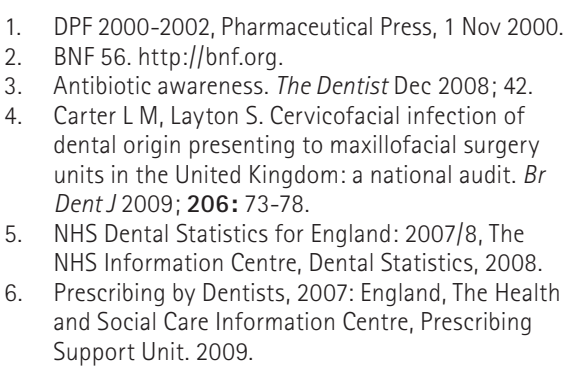

5. NHS Dental Statistics for England: 2007/8, The NHS Information Centre, Dental Statistics, 2008.

6. Prescribing by Dentists, 2007: England, The Health and Social Care Information Centre, Prescribing Support Unit. 2009.

DOI: $10.1038 /$ sj.bdj.2009.525

\section{SHADE GUIDES}

Sir, I have enjoyed reading the first of $\mathrm{Dr}$ Ahmad's series on Digital dental photography. The first dental image shown is a photograph of teeth and a shade guide together to assist a technician who is not present. This is an excellent, almost essential procedure for the majority of dentists who work with a distant technician.

Unfortunately, as Gordon Christensen illustrated in the CRA newsletter of May 2005, the method Dr Ahmad shows reflects a common error as the shade guide is not in the same plane as the teeth. A preferred method is for a single selected shade tab to be inside the oral cavity, so that the illumination for shade tab and teeth is the same.

When the technique Dr Ahmad shows is used, the shade guide will normally be significantly lighter than the teeth.

\section{J. Reuter, Latimer}

DOI: 10.1038/sj.bdj.2009.526 